Communications in Physics, Vol. 29, No. 3 (2019), pp. 197-214

DOI:10.15625/0868-3166/29/3/13733

\title{
NONLINEAR OPTICAL TWEEZERS AS AN OPTICAL METHOD FOR CONTROLLING PARTICLES WITH HIGH TRAP EFFICIENCY
}

\author{
HO QUANG QUY ${ }^{a, b, \dagger}$ \\ ${ }^{a}$ Ho Chi Minh University of Food Industry, 140 Le Trong Tan, Tan Phu, Ho Chi Minh City \\ ${ }^{b}$ Academy of Military Science and Technology, 17 Hoang Sam, Cau Giay, Hanoi \\ ${ }^{\dagger}$ E-mail: linhson8294@gmail.com
}

Received 5 April 2019

Accepted for publication 19 June 2019

Published 15 August 2019

\begin{abstract}
Optical tweezers have been seen as an essential tool for manipulating dielectric microparticles and nanoparticles thanks to its non-contact action and high resolution of optical force. Up to now, there has been a lot of optical tweezers applications in the fields of biophysics, chemistry, medical science and nanoscience. Recently, optical tweezers have been theoretically and experimentally developed for the nano mechanical characterization of various kinds of biological cells. The configuration of optical tweezers has been day after day improved to enhance the trapping efficiency, spatial and temporal resolution as well as to ease the control of trapped objects. In common trend of optical tweezers improvements, we will discuss in detail several configurations of nonlinear optical tweezers using nonlinear materials as the added lens. We will also address the advantages of nonlinear optical tweezers, such as enhancement of optical efficiency, reduction of trapping region, and simplification in controlling all-optical method. Finally, we will present discussions about the specific properties of nonlinear optical tweezers used for stretch DNA molecule as example and an idea to improve nonlinear optical tweezers using thin layer of organic dye proposed for going time.
\end{abstract}

Keywords: nonlinear physics; biophysics; optical tweezers, nonlinear optical tweezers, DNA molecule.

Classification numbers: 42.65.Wi; 42.79.-e; 87.80.-y.

(C)2019 Vietnam Academy of Science and Technology 


\section{INTRODUCTION}

Up to now, the optical tweezers (OT) has been becoming an efficient support tool widely applied in physics, chemistry, medical science, nanoscience and biology [1,2]. The idea of OT was first reported in 1970 by Arthur Ashkin [3], a former Bell Laboratories researcher awarded the 2018 Nobel Prize in Physics "for the optical tweezers and their application to biological systems" on October $2^{\text {nd }}, 2018$. Two main parts of OT are the laser source and high numerical aperture (NA) microscope objective creating a laser beam with high spatial gradient of intensity, i.e., the Gaussian beam, commonly. Irradiated by the Gaussian beam, the dielectric particle having refractive index $n_{p}$ larger than that of its surrounding fluid $n_{s f}\left(m=n_{p} / n_{s f}>1\right)$ should be pulled into the center of beam waist. Meanwhile, irradiated by the hollow Gaussian beam, the dielectric particle with refractive index smaller than that of its surrounding fluid $\left(m=n_{p} / n_{s f}<1\right)$ should be pulled into the center of dark region. So the optical tweezers are used to trap and hold the dielectric particles, and to manipulate (or to control) them in space of embedding fluid [4-6]. For the diversified applications $[7,8]$ there is a lot of methods used to trap and control trapped dielectric particles as: laser beam scanning used rotation system of mirrors [1], laser beam scanning used acousto-optical deflector [9], focused laser controller using intelligent electro-mechanical or opto-mechanical system with help of the computer, etc. [4,10-12]. To control trapped dielectric particles in 2D or 3D space, all mentioned methods need two facts, at least [13]. Lately, to avoid the complexity of opto-mechanical system, an all-optical method to control dielectric particles in nonlinear embedding fluid by tuning of laser power is proposed [6] based on the Kerr effect [14-16]. However, this proposal for optical tweezers meets a difficulty that the embedding fluid could not immediately change suitable to other dielectric particles due to ratio of their refractive indexes. Referring to idea of this method, the acousto-optical tweezers whose operation is based on the nonlinear response of refractive index of acousto-optical material to intensity of the acoustic wave, are proposed and investigated to control dielectric particles in 2D or 3D space of embedding fluid [17]. Although our proposals could be really used to design optical tweezers, they need a high intensity of acoustic wave, which is the difficult problem in experiment.

As well-known, since the nonlinearity of convenient fluid and interesting particles are very low, for example the nonlinear refractive index of water, polystyrene and silica is just $n_{w}=$ $2.7 \times 10^{-20} \mathrm{~m}^{2} / W[18], n_{p}=5.9 \times 10^{-17} \mathrm{~m}^{2} / W$ [19] and $n_{s}=2.0 \times 10^{-20} \mathrm{~m}^{2} / \mathrm{W}$ [20], respectively, so the nonlinear effect in OT should be observed only if using the high-repetition ultrafast pulsed laser [21-23]. Consequently, the nonlinear effect is very weak and then practically to enhance optical trap efficiency (OTE) it needs a high laser intensity [6]. Fortunately, there is a lot of organic dyes with high nonlinearity as Diclothane Polimetin [24] with $n_{D p} \approx 5.5 \times 10^{-8} \mathrm{~cm}^{2} / \mathrm{W}$, Acid blue with $n_{A b} \approx 1 \times 10^{-10} \mathrm{~m}^{2} / \mathrm{W}$ [25], Mercurochrome with $n_{M e r} \approx 1 \times 10^{-11} \mathrm{~m}^{2} / \mathrm{W}$ at laser wavelength of $532 \mathrm{~nm}$ [26], and Acid Green with $n_{A g} \approx 1 \times 10^{-11} \mathrm{~m}^{2} / W$ at laser wavelength of $635 \mathrm{~nm}$ [27]. All mentioned organic dyes are not only in solvent, but can be also chemically accumulated in the glass plate with thickness below millimeter, which has been used to observe the nonlinear refraction as well as laser limiter [28]. The high nonlinearity organic dyes have given us an opportunityto design a compact nonlinear optical tweezers (NOTOD), in which a thin layer of organic dye combined with the microscope objective replaces the nonlinear surrounding fluid to play the nonlinear lens $[14,15]$. Proposed NOTOD can be used to longitudinally control particles embedded in the linear surrounding fluid chemically suitable to the bio-subjects [29]. Using NOTOD, 
the OTE is remarkably enhanced in comparison with the convenient linear OT [30]. Moreover, by NOTOD the trapped particles will be controlled not only by all-optical method [31], but the difficulties of choice of the nonlinear surrounding fluid chemically suitable to bio-subjective as the living cell can also be overcome.

In this paper, we present the usefulness of Kerr effect in optical trap, relative theoretical analysis and some of NOT's configurations in progress. Then, we numerically investigate and discuss on some advantages of NOT for the control trapped and stretch DNA molecule by tuning laser power, and the configuration of NOTOD suitable to the different kind of DNA molecules.

\section{TRAP PRINCIPLES}

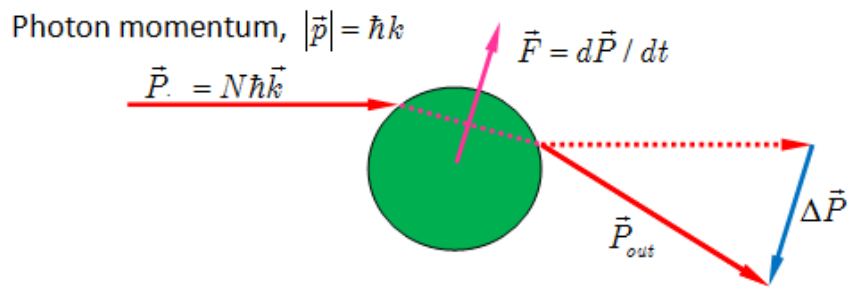

Fig. 1. Optical force due to photons meeting a refracting object.

Let us consider a micro-particle irradiated by the laser beam consisting of $N$ photons which have the momentum $\vec{p}=\hbar \vec{k}$. After interacting with the particle, the momentum of photons, $\vec{P}_{i n}=$ $N \hbar \vec{k}$ is changed to $\vec{P}_{\text {out }}$. Because of the conservation of momentum, the particle will receive a momentum from the photons $\Delta \vec{P}=\vec{P}_{\text {in }}-\vec{P}_{\text {out }}$ and then moves under optical pressure or optical forces $\vec{F}=d \vec{P} / d t$ following the third law of motion by Isaac Newton (Fig. 1). If all optical forces are in homogeneous balance, the object will be hold at stable site $[1,3,4]$. The motion direction of particle depends on the kind of acting optical forces. If a Gaussian laser beam with intensity distribution as the function given as [30]:

$$
I(\rho, z)=\frac{I_{0}}{1+\left(z / z_{0}\right)^{2}} \exp \left(-\frac{\rho^{2}}{W_{0}^{2}\left(1+\left(z / z_{0}\right)^{2}\right)}\right)
$$

where $I_{0}$ is peak intensity at center beam waist, $(\rho=0, z=0), W_{0}$ is the beam waist radius, $z_{0}$ is the Rayleigh distance, acting on the particle with radius $a$, refractive index ratio $m>1$, there are three forces [32]:

i) axial gradient force,

$$
\vec{F}_{g r d, z}=-\hat{z} \alpha \nabla_{z} I(\rho, z)=-\hat{z} \alpha \frac{2 z}{z_{0}\left[1+\left(\frac{z}{z_{0}}\right)^{2}\right]}\left\{\frac{\rho^{2}}{W_{0}^{2}\left[1+\left(\frac{z}{z_{0}}\right)^{2}\right]}-1\right\} I(\rho, z),
$$


ii) radial gradient force,

$$
\vec{F}_{g r d, \rho}=-\hat{\rho} \alpha \nabla_{\rho} I(\rho, z)=-\hat{\rho} \alpha\left\{\frac{2 \rho}{W_{0}^{2}\left[1+\left(\frac{z}{z_{0}}\right)^{2}\right]}\right\} I(\rho, z),
$$

iii) and axial scattering force,

$$
\vec{F}_{s c t, z}=\hat{z} \beta I(\rho, z)
$$

where $\hat{\rho}, \hat{z}$ are unit vectors in radial and axial directions, respectively, $\alpha=2 \pi n_{f l} a^{3}\left(\frac{m^{2}-1}{m^{2}+2}\right)$ is generalized polarity of particle, $\beta=\frac{128 \pi^{5} a^{3}}{3 \lambda^{4}}\left(\frac{m^{2}-1}{m^{2}+2}\right)^{2}$ is scattering coefficient [3]. From Eqs. (2) - (4), we can see that the particle moves always in the direction opposite to that of gradient forces, and in the same direction as that of scattering force. The motion of particle under optical forces is illustrated in Fig. 2 a,b and trap principle is given in Fig. 2c. In the case $m<1$, i.e., $\left(m^{2}-1\right) /\left(m^{2}+2\right)<1$, it is necessary that $\nabla_{\rho} I(\rho, z)<1$, i.e., the hollow Gaussian beam will be used. Since the scattering force is very small neglectable in comparison with gradient forces [3], so the total force, $F_{t o l}$ pulls always to the center of beam waist, and the particle is trapped at stable site, where the optical total forces are symmetrically directed and in balance. The stable degree of trapped particle or stiffness of optical tweezers depends on the magnitude of force peaks [33] and the spatial distance between them [34], i.e., depends on height and diameter of the distribution of $\left|F_{g r d, \rho(g r d, z)}\right|$, which is similar to the OT's potential bell, $\left\langle U_{\rho(z)}\right\rangle=-\left\langle P_{\rho(z)} \cdot E\right\rangle$, where $P_{\rho(z)}$ is the radial (or axial) polarization and $E$ is the electric field of laser (Fig. 1d) [21].

From Eqs. (1)-(4) and Figs. 2a, 2b, it is easy to show that the gradient force reaches peak at $|\rho|=W_{0}$ (or $|z|=z_{0}=\pi W_{0}^{2} / \lambda$ ) and the magnitude of peaks is proportional to the radius of particle $\left(a^{3}\right)$, refractive index ratio $(m)$ and gradient of intensity $(\nabla I)$. That means, if the radius of particle and refractive index of surrounding fluid are given, the increase of particle's index $\left(n_{p}\right)$ and of peak intensity $\left(I_{0}\right)$ leads to the increase of the peak of $\left|F_{\text {grd }, \rho(g r d, z)}\right|$, meanwhile, the decrease of beam waist's radius $\left(W_{0}\right)$ as well as of Rayleigh distance $\left(z_{0}\right)$ leads to the decrease of the distance between peaks.

So, to enhance the stable degree of particle in OT, it is necessary to increase peak intensity of laser, refractive index of particle, and decrease beam waist's radius. By that way, not only the stable degree of particle is enhanced, but also the OTE [30]

$$
Q=\frac{F c}{n_{s f} P}
$$

with $P$ the laser beam average power and $c$ is the light velocity, increases [35]. The Kerr effect seems to be a fact to be used to enhance OTE, so in the next section, its influence on the properties of OT will be discussed. 

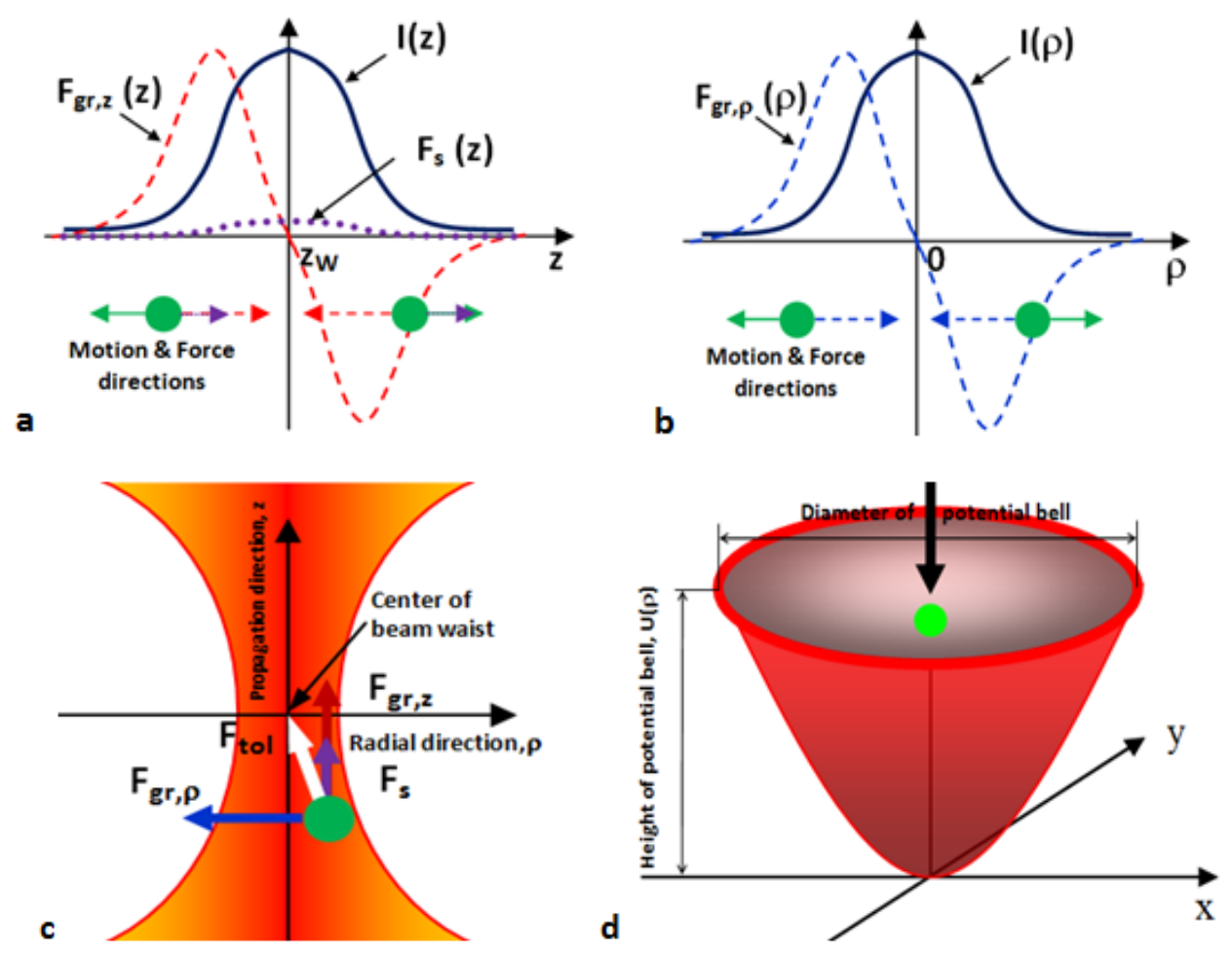

c

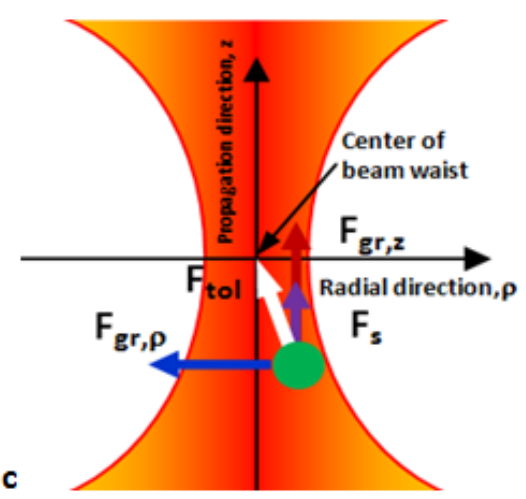

Fig. 2. Illustration of trap principle: a) Axial forces act on particle, b) Radial force acts on particle, c) Total force pulls particle into trap site, d) Radial potential bell.

\section{OTE OF OT FOR KERR MICROSPHERES}

Consider a Kerr particle embedded in the linear surrounding fluid. Then, the refractive index ratio will satisfy the following

$$
m_{n l}(\rho, z)=\frac{n_{p}+n_{2} I(\rho, z)}{n_{s f}}
$$

where $n_{2}$ is the nonlinear coefficient of refractive index of Kerr particle. From Eqs. (2), (3), (5) and (6), it is easy to have the ratio of OTEs, $\eta$

$$
\eta=\frac{Q_{n l}}{Q}=\frac{F_{n l}}{F}=\frac{\left(m_{n l}^{2}-1\right)}{\left(m_{n l}^{2}+2\right)} / \frac{\left(m^{2}-1\right)}{\left(m^{2}+2\right)}
$$

where $F_{n l}, Q_{n l}$ are the gradient force acting on Kerr particle and relating OTE, respectively. From Eqs. (6) and (7), it is clear that the efficiency enhancement (EE) defined as $E_{\text {eff }}=10 \times \log (\eta)$ depends on the nonlinear coefficient of refractive index and laser intensity. This conclusion is proved in Fig. 3 plotted with given parameters: $n_{s f}=1.45, n_{p}=1.5$, i.e., $m>1$ (a) and $n_{p}=1.3$, i.e., $m<1$ (b). We can see that in the case $m>1$ (Fig. 3a), the EE increases with increasing of both facts, laser peak intensity and nonlinearity of particle, but it has an asymptote value of $16.42 \mathrm{~dB}$. This value is corresponding to the maximum value of ratio $\left(m_{n l}^{2}-1\right) /\left(m_{n l}^{2}+2\right)=1$. 
For the $m<1$ (Fig. 3b), the EE-peak intensity characteristics are plotted forpurpose to compare with the case of $m=n_{p} / n_{s f}=1.5 / 1.45>1$. We can see that the optical trap is not efficient till $n_{p}+n_{2} I=n_{s f}$ and then the EE increases with an increasing peak intensity and nonlinearity of particle. Consequently, the OTE is remarkably enhanced for the Kerr particle, even for the particle with $m<1$ by tweezers using pulsed Gaussian beam with high intensity [21,22]. That means, it is not necessary to use Hollow-Gaussian beam to trap Kerr particle with $m<1$ till the laser intensity satisfying the condition $I_{0} \geq\left(n_{s f}-n_{p}\right) / n_{2}[15]$.

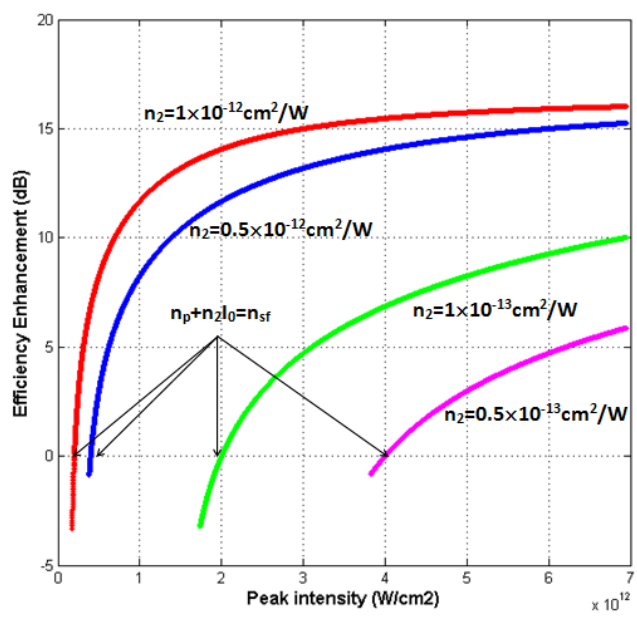

(a)

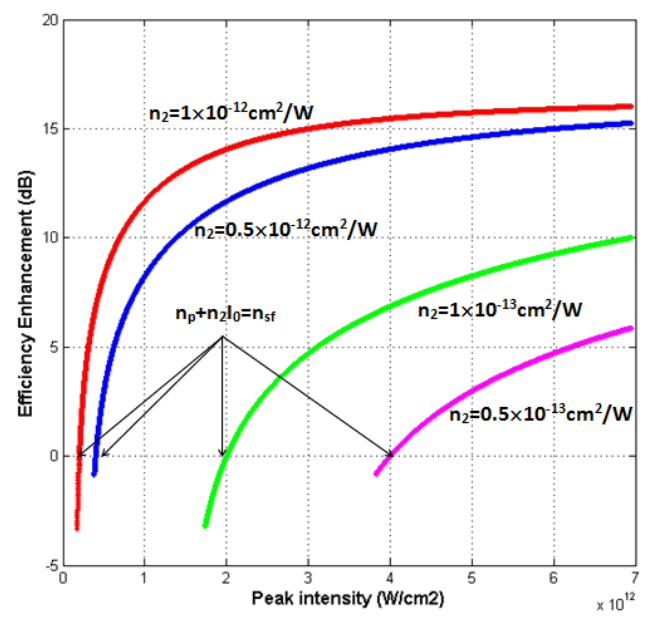

(b)

Fig. 3. $E_{\text {eff }}$ vs. $I_{0}$ with different $n_{2}$. a): $m>1$ and b): $m<1$.

\section{NOT USING KERR EMBEDDING FLUID}

Now, we consider a linear particle with $n_{p}=1.5$ embedded in the thin nonlinear surrounding fluid with refractive index of $1.45+n_{2} I$ [15]. In this case, the index ratio is modified as:

$$
m_{n l}(\rho, z)=\frac{n_{p}}{n_{s f}+n_{2} I(\rho, z)}
$$

Using Eqs. (7) and (8), the EE-peak intensity characteristics are numerically calculated and presented in Fig. 4. Form Fig. 4a, we can see that the EE reduces with increasing of peak intensity and nonlinearity of surrounding fluid. That means, in the surrounding fluid with low nonlinearity, the Kerr effect can be neglected. The Kerr effect can be neglected also if the nonlinearity of particle is larger than that of surrounding fluid, since in this case the EE still rises up (Fig. 4b). It is clear that using Kerr surrounding fluid in OT can reduce the OTE of OT using Gaussian beam. However, a Kerr surrounding fluid having thickness many times larger than the particle size in OT can play an important role of the optical method to control the particle in space $[6,36]$. As we know, a nonlinear medium irradiated by the intense laser Gaussian beam can be the nonlinear lens [37]. Consequently, the laser beam will be reshaped by the nonlinear lens as shown in Fig. 5 . Consider an input Gaussian beam with intensity distribution given in Eq. (1) that irradiates the 


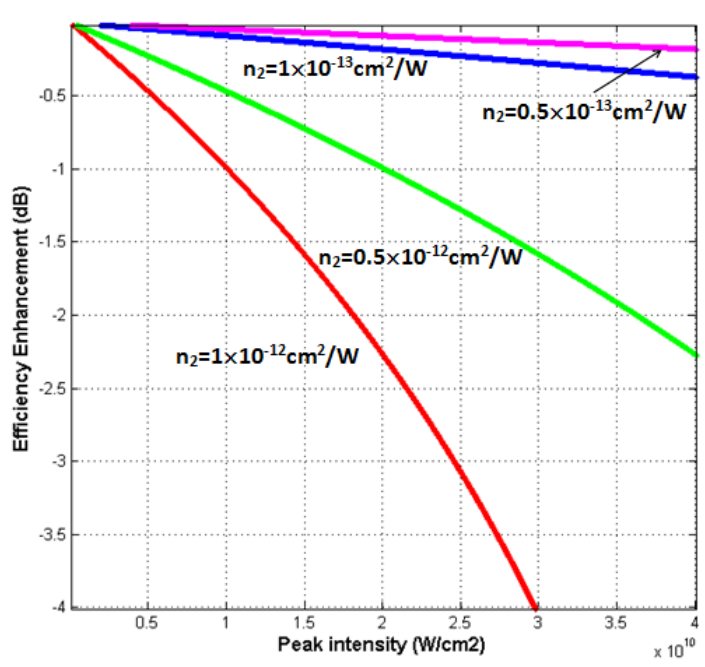

(a)

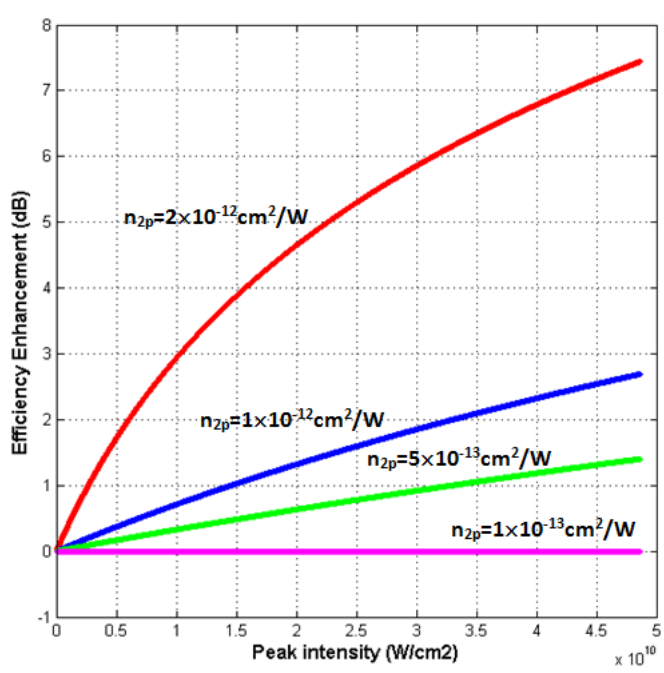

(b)

Fig. 4. (a) $E_{\text {eff }}$ vs. $I_{0}$ with different $n_{2}$ of surrounding fluid; (b) $E_{\text {eff }}$ vs. $I_{0}$ with different $n_{2 p}$ of particle and fixed $n_{2 s f}$ of surrounding fluid.

Kerr medium with thickness of $d$ (Fig. 5). Using the propagation matrix [37] of light through optical system consisting of $m$ thin plates with thickness $\Delta z=d / m$ and Kerr relation in Eq. (8), the intensity redistribution of laser beam propagating through the $\mathrm{i}^{\text {th }}$ plate is derived as follows

$$
I_{m, i}(\rho, z)=I_{0} \times \frac{1}{1+\left[\frac{z+\left(z_{i}+\Delta z\right)}{z_{0, i}}\right]^{2}} \times \exp \left[-\frac{\rho^{2}}{W_{0, i}^{2}} \frac{1}{1+\left[\frac{z+\left(z_{i}+\Delta z\right)}{z_{0, i}}\right]^{2}}\right]
$$

where

$$
W_{0, i}=M_{i} W_{0,(i-1)}, \quad z_{i}=M_{i}^{2}\left(\Delta z-f_{n l, i}\right)+f_{n l, i}, \quad z_{0, i}=M_{i}^{2} z_{0,(i-1)}
$$

are the radius of beam waist, waist position and Rayleigh distance, respectively. $M_{i}=M_{r, i} / \sqrt{1+r_{i}^{2}}$, $r_{i}=z_{(i-1)} /\left(\Delta z-f_{n l,(i-1)}\right), M_{r, i}=\left|f_{n l, i} / \Delta z-f_{n l, i}\right|$, and

$$
f_{n l, i}=\frac{\left[1+\left(\Delta z / z_{0,(i-1)}\right)^{2}\right]^{2}}{2 n_{n l} I_{0} \Delta z}
$$

is the focal length of $\mathrm{i}^{\text {th }}$ nonlinear lens.

We have observed the reshaping of an input Gaussian beam with wavelength of $\lambda=1.06 \mu \mathrm{m}$, peak intensity of $I_{0}=3.5 \times 10^{8} \mathrm{~W} / \mathrm{cm}^{2}$ and beam waist of $W_{0}=2 \mu \mathrm{m}$ located at distance $d=$ $-10 \mu \mathrm{m}$ from input surface of Kerr medium. Its intensity distribution in phase plane $(\rho, z)$ is illustrated in Fig. 6a. Using Eqs. (9) - (11), the intensity distributions of modified Gaussian beam through Kerr medium with $n_{s f}=1.45, n_{2 s f}=1 \times 10^{-10} \mathrm{~cm}^{2} / \mathrm{W}$ and $n_{2 s f}=2 \times 10^{-10} \mathrm{~cm}^{2} / \mathrm{W}$ are cascade simulated and shown in Fig. 6b and Fig. 6c, respectively. We can see that when the nonlinearity of Kerr medium increases, the radius of beam waist reduces from 2 through 1.8 to 


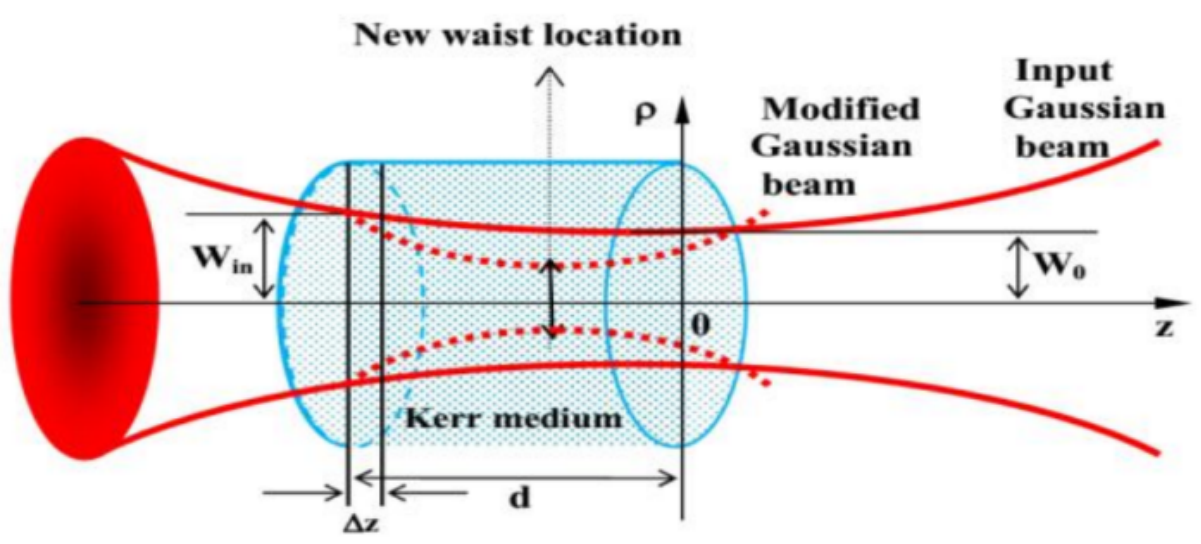

Fig. 5. Sketch of NOT with thick Kerr medium using Gaussian beam [36].

$1.4 \mu \mathrm{m}$, while its position moves close to the input surface of Kerr medium from 10 through 6.82 to $3.82 \mu \mathrm{m}$. Substituting Eq. (9) to Eq. (2) we have the axial gradient force as follows [14]:

$$
\vec{F}_{g r d, z}(\rho, z)=-\hat{z} \frac{2 \beta_{n, i}(\rho, z) I_{m, i}(\rho, z)}{n_{m, i}(\rho, z) \varepsilon_{0} c k W_{0, i}} \frac{1}{\left(1+\left(\frac{z+\left(z_{i}+\Delta z\right)}{z_{0, i}}\right)^{2}\right)^{2}} \times \frac{z+\left(z_{i}+\Delta z\right)}{z_{0, i}^{2}}
$$

where $\beta_{n, i}(\rho, z)$ is the polarity and $n_{m, i}(\rho, z)$ is the total index of Kerr medium relating to $I_{m, i}(\rho, z)$.

The distribution of axial gradient force acting on the dielectric particle $\left(a=200 \mathrm{~nm}, n_{p}=\right.$ $1.5)$ is changed, with different value of nonlinearity of Kerr medium. The distributions of $F_{g r d, z}(\rho, z)$ for $n_{2 s f}=0, n_{2 s f}=1 \times 10^{-10} \mathrm{~cm}^{2} / \mathrm{W}, n_{2 s f}=2 \times 10^{-10} \mathrm{~cm}^{2} / \mathrm{W}$ are presented in Fig. 6d, Fig. 6e and Fig. 6f, respectively. We can see that the trap site, where $F_{g r d, z}(\rho, z)=0$, moves close to the input surface with increasing of nonlinearity of medium. The dependence of the trap site, where particle is stably trapped in Kerr medium on the laser intensity, gives us an idea to tune laser intensity for controlling trapped particle in the space. A NOT using two laser sources used to control trapped bead linked to ADN molecule is investigated [6]. In the configuration presented in Fig. 7a, a weak laser is used to control the radial position (stable position, $\rho_{s t}$ ) of trapped linked to DNA molecule (Fig. 7b), and an intense one is used to control the axial position (stable position, $z_{s t}$ ). The motion of trapped bead linked to DNA molecule in Kerr medium, under optical forces and elastic force can be theoretically observed by using the general Langevin equation [38]:

$$
m \ddot{\rho}(t)=-\gamma \dot{\rho}(t)+F_{g r d, \rho}(\rho(t))-F_{e l}(\rho(t))+\sqrt{2 k_{B} T \gamma} W_{\rho}(t)
$$

where $m$ is the bead mass; $\gamma=6 \pi \eta a$ is the friction coefficient; $\eta$ is the viscosity of surrounding fluid, $a$ is the radius of bead, $k_{B}$ is Boltzmann constant, $T$ is the temperature, $W_{\rho}(t)$ is the white noise, $\rho$ is the bead's radial position (Fig. 7b), the last three terms in the right are the radial optical gradient, elastic and Brownian forces, respectively. The elastic force of DNA molecule is given as follows [39,40]:

$$
F_{e l}(\rho)=\frac{k_{B} T}{L_{b}}\left[\frac{\rho-\rho_{0}}{L}+\frac{1}{4\left(1-\left(\rho_{-} \rho_{0}\right) / L\right)^{2}}-\frac{1}{4}\right]
$$




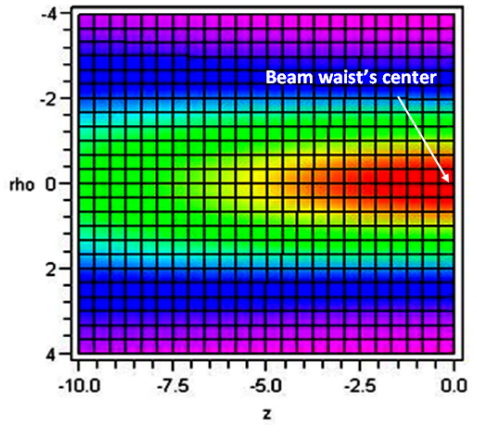

(a)

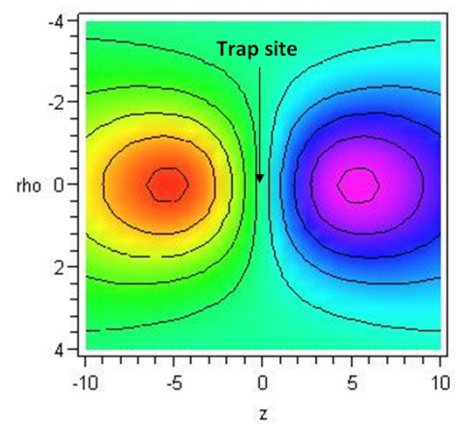

(d)

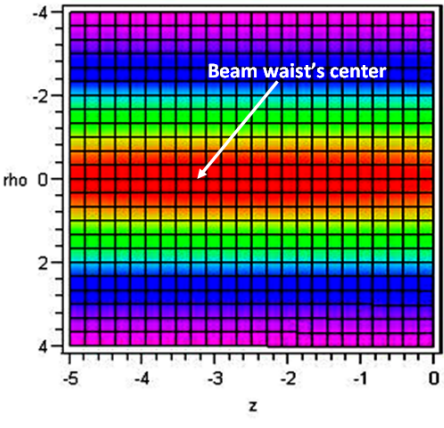

(b)

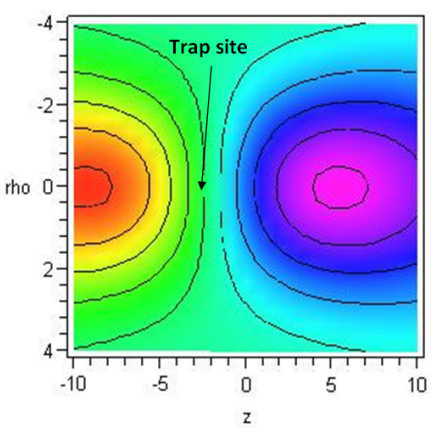

(e)

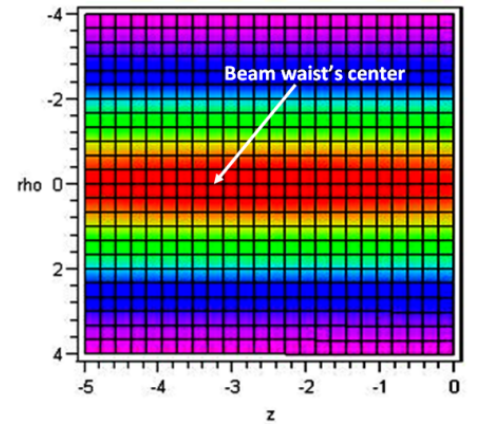

(c)

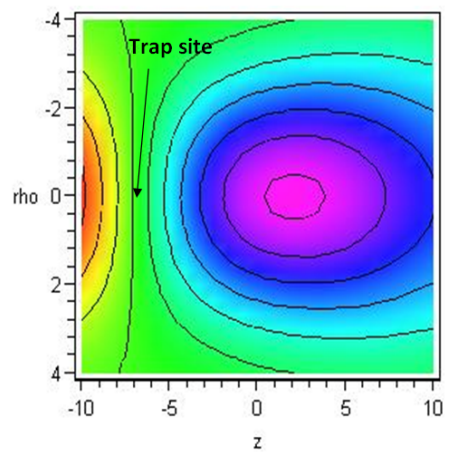

(f)

Fig. 6. Intensity (a), (b), (c) and axial gradient force (d), (e), (f) distributions in phase plane $(\rho, z)$ of Kerr medium.

where $L_{b}, L$ are the persistence and contour lengths of DNA molecule, respectively, $\rho_{0}$ is the begin position of trapped bead (and of anchor's position). Using the experimental parameters as given in Ref. [6], the radial position (Fig. 7c) and axial position-average power characteristics (Fig. 7d) are numerically plotted for polystyrene bead [38] linked to $\lambda$-phage DNA molecule [39].

Under the action of the radial optical force the trapped bead speedily moves close to laser axis, and it reaches to the stable site till the optical force and the elastic force of DNA molecule are in balance. Based on this phenomenon, the stable position of trapped bead in radial direction can be controlled by tune of weak laser power (Fig. 7c). This investigated controlling method is absolutely similar to using a convenient OT using the linear surrounding fluid [1]. In some cases for that the contour length of DNA molecule is too long, meanwhile the begin position of trapped bead is close to the laser axis, the stretched length does not reach to the extreme by this method [40]. Though the trapped bead can be held in the stable position, the stretched length of DNA has not reached to the extreme. It is necessary to move this site along the laser axis. For this purpose, the efficient way is to use the intense laser. As shown in Fig. 5, by tune of intense laser power, the stable position moves in axial direction (Fig. 7d). From Fig. 7d, if the power of intense laser changes an interval of $2.5 \mathrm{~kW}$, the stable position can change an interval of $40 \mu \mathrm{m}$, 


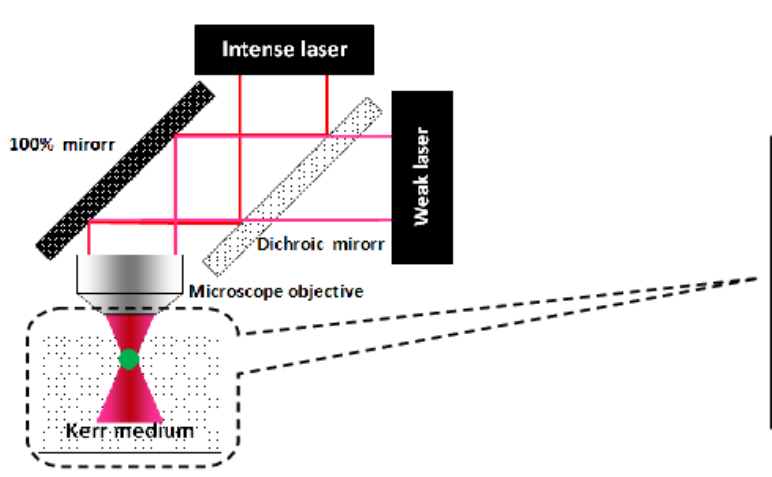

(a)

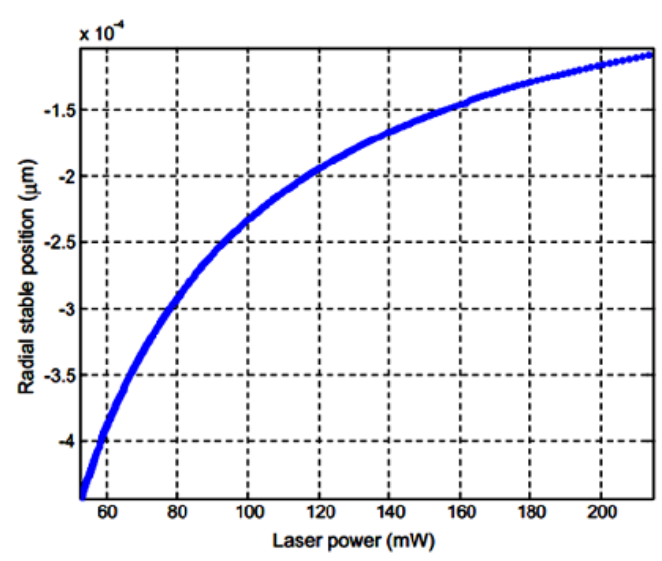

(c)

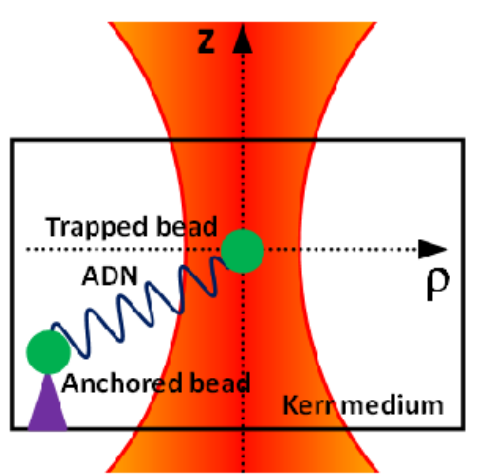

(b)

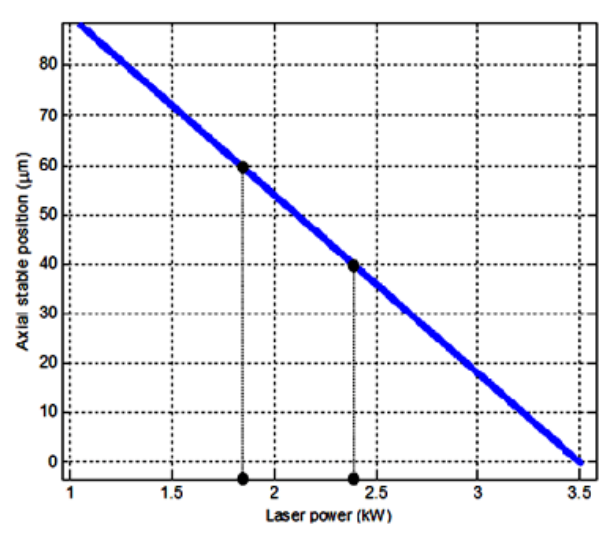

(d)

Fig. 7. Sketch of NOT using two lasers; b) DNA with two bead in Kerr medium; c) Radial position vs. weak laser power [6]; d) Axial position vs. intense laser power [6].

so the proposal NOT can longitudinally control any DNA molecules with contour length shorter than $40 \mu \mathrm{m}$ linked to trapped bead located on the axis of intense laser.

\section{OT USING THIN LAYER OF ORGANIC DYE (NOTOD)}

As shown above, the NOT using Kerr medium (Fig. 7a) can be used as an all-optical method to control particle in 3D space. However there are two weak points: the first one is the necessity of high laser intensity, which can biophysically damage the trapped bead, and the second one belongs to biochemical properties, the Kerr medium is not always suitable to different kinds of trapped bead and DNA molecule. To pass those complexities, a NOTOD using Kerr medium with the high nonlinearity as added nonlinear lens has been proposed [29,30]. The configuration of NOTOD using the organic dye Acid Blueis presented in Fig. 8a. The difference between the conventional OT and NOTOD is that the high NA microscope objective is replaced by an 
optical system consisting of a low NA objective and thin layer of Acid Blue. Acid blue is an organic dye which has nonlinearity of $n_{2} \sim 1 \times 10^{-6} \mathrm{~cm}^{2} / \mathrm{W}$, which is much higher than convenient liquids [41]. Irradiated by the intense Gaussian beam (as Eq. (1)), the layer of Acid blue can be a nonlinear lens with the nonlinear focal length given by [29]:

$$
f_{n l}=\frac{W_{0}^{2}}{d n_{2} I_{0}}
$$

where $d$ is the thickness of layer.

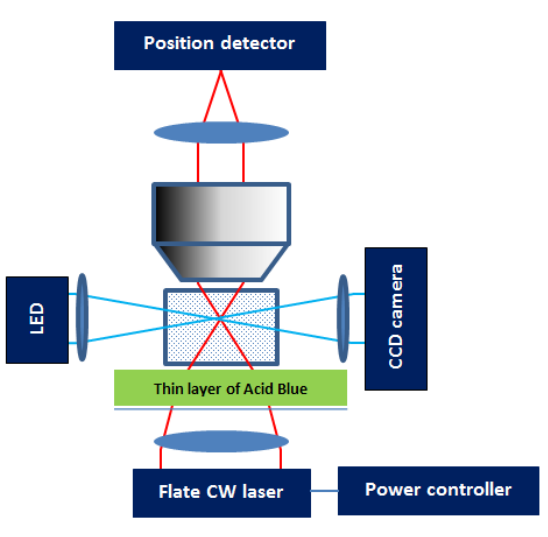

(a)

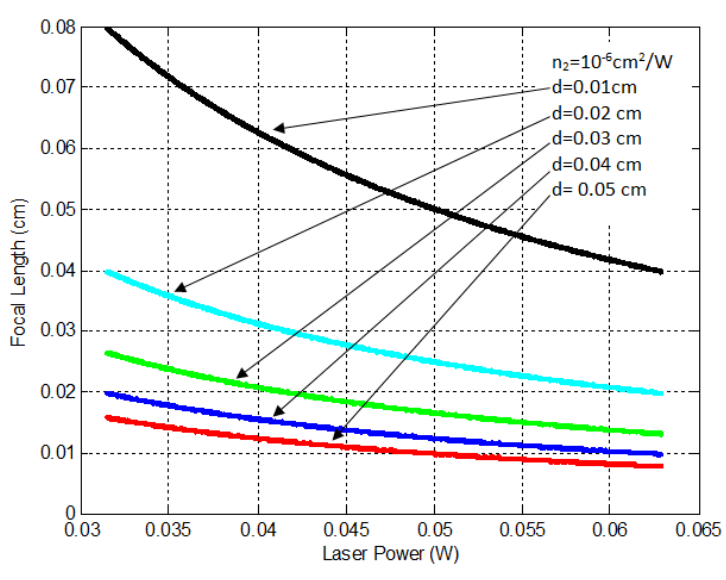

(b)

Fig. 8. a) Sketch of NOTOD using thin layer of Acid Blue; b) Focal length of thin layer with different thickness vs. average power of incoming Gaussian laser beam, and $W_{0}=$ $0.002 \mathrm{~cm}$.

Consider a plate CW beam focused by a low NA objective to Gaussian beam with waist of $W_{0}=0.002 \mathrm{~cm}$ which is about 20 times larger than the laser spot which is for conventional TO (about $2 \mu \mathrm{m}$ ) irradiating a thin layer of Acid blue. Because of high intensity of incoming Gaussian beam, the thin layer of Acid Blue becomes nonlinear lens, which has focal length $f_{n l}$ depending on an average power of laser and thickness of Acid blue layer (Fig. 8b). It is clear that the nonlinear focal length is shorter when the laser power is higher and the layer is thicker. It not only becomes the nonlinear lens, but also in return reshapes the incoming laser beam whose intensity distribution on plane $(\rho, z)$ is given as follows [29]:

$$
I(\rho, z)=\frac{I_{0}}{1+\left(\frac{z \pi\left(d n_{2} I_{0}\right)^{2}}{\lambda W_{0}^{2}}\right)^{2}} \exp \left(-\frac{\rho^{2}}{\left(\frac{\lambda W_{0}}{\pi d n_{2} I_{0}}\right)^{2}\left[1+\left(\frac{z \pi\left(d n_{2} I_{0}\right)^{2}}{\lambda W_{0}^{2}}\right)^{2}\right]}\right)
$$

Using Eq. (16) and choosing $d=0.05 \mathrm{~cm}$, the intensity distribution of reshaped laser beam in phase plane $\left(d=-2 W_{0}^{\prime} \div 2 W_{0}^{\prime}, z=-f_{n l} \div f_{n l}\right)$, where $W_{0}^{\prime}=\lambda W_{0} / \pi d n_{2} I_{0}$ is the waist's radius, is numerically observed with different average power of incoming beam and presented in Fig. 9. 


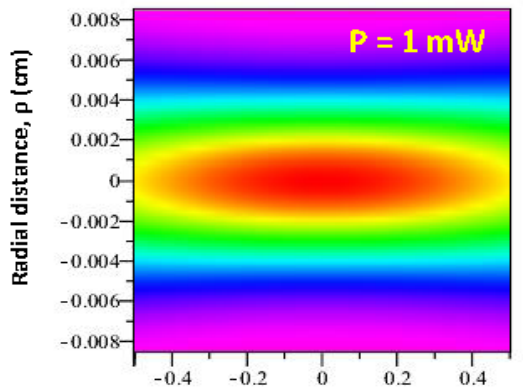

Distance from focus, $\mathrm{z}(\mathrm{cm})$

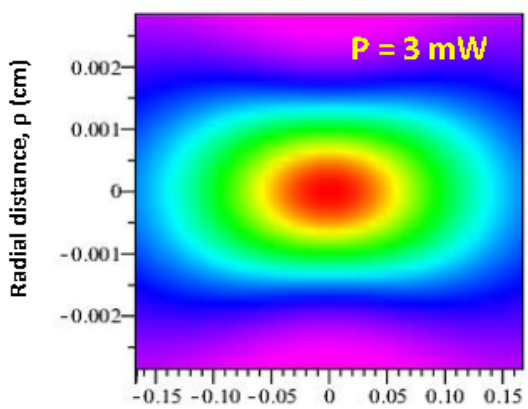

Distance from focus, $\mathrm{z}(\mathrm{cm})$

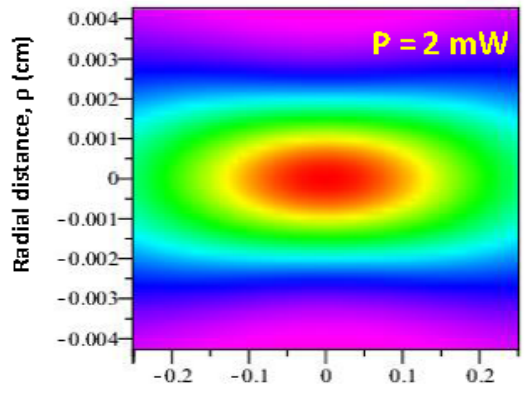

Distance from focus, $\mathrm{z}(\mathrm{cm})$

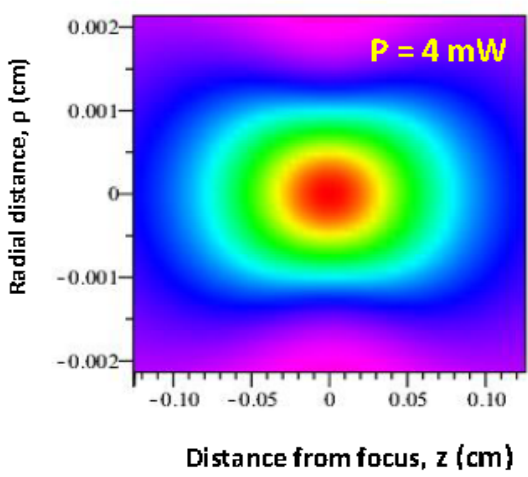

Fig. 9. Distribution of intensity of reshaped laser beam in phase plane $(\rho, z)$ with different average power of incoming laser [31].

We can see that the radius of reshaped laser beam $W_{0}^{\prime}$ and the focal length of nonlinear lens $f_{n l}$ are decreased when the average power of incoming laser beam $P=\pi W_{0}^{2} I_{0} / 2$ increases, consequently, intensity is more and more enhanced around the focus. The important point here is that the intensity gradient enhances with increasing of average power. Using Eqs. (2), (4) and (16), the total axial force acting on the dielectric particles is given as follows:

$$
\begin{aligned}
& \vec{F}_{\text {total }, z}(\rho, z) \\
&=\hat{z}\left\{-2 \pi n_{f l} a^{3}\left(\frac{\left(\frac{n_{b}}{n_{f l}}\right)^{2}-1}{\left(\frac{n_{b}}{n_{f l}}\right)^{2}+2}\right) \frac{2 z}{\left(\frac{\pi\left(d n_{2} I_{0}\right)^{2}}{\lambda W_{0}^{2}}\right)\left\{1+\left(\frac{z \pi\left(d n_{2} I_{0}\right)^{2}}{\lambda W_{0}^{2}}\right)^{2}\right\}^{2}}\left(\frac{\rho^{2}}{\left(\frac{\lambda W_{0}}{\pi d n_{2} I_{0}}\right)^{2}\left\{1+\left(\frac{z \pi\left(d n_{2} I_{0}\right)^{2}}{\lambda W_{0}^{2}}\right)^{2}\right\}}-1\right)\right. \\
&\left.+\frac{128 \pi^{5} a^{3}}{3 \lambda^{4}}\left(\frac{\left(\frac{n_{b}}{n_{f l}}\right)^{2}-1}{\left(\frac{n_{b}}{n_{f l}}\right)^{2}+2}\right)^{2}\right\} \times I_{0} \exp \left[-\frac{\rho^{2}}{\left(\frac{\lambda W_{0}}{\pi d n_{2} I_{0}}\right)^{2}\left\{1+\left(\frac{z \pi\left(d n_{2} I_{0}\right)^{2}}{\lambda W_{0}^{2}}\right)^{2}\right\}}\right] .
\end{aligned}
$$

Using parameters given above to Eq. (17), the distribution of total axial force along laser beam axis is numerically plotted in Fig. 10. From this figure we can see: first, when the average 
power is tuned an amount of $\Delta P=3 \mathrm{~mW}$, the trap center will move a distance of $\Delta z_{t c}=0.38 \mathrm{~cm}$; second, with an increasing of the average power the distance between two peaks which is called trap region, $\Delta z_{s t}$ [34] reduces and the peak force increases. The second point shows that the OTE increases. The enhancement of OTE of NOTOD in comparison to that of linear optical tweezers (LOT) using Gaussian beam with average power of $P=1 \mathrm{~mW}$, and $W_{0}=2 \mu \mathrm{m}$ is observed in Fig. 10. The curves in Fig. 11 are plotted for $Q_{\rho, \max }=F_{g r d, \rho}^{\text {peak }} c / n_{s f} P$ and $Q_{z, \max }=F_{g r d, z}^{\text {peak }} c / n_{s f} P$.

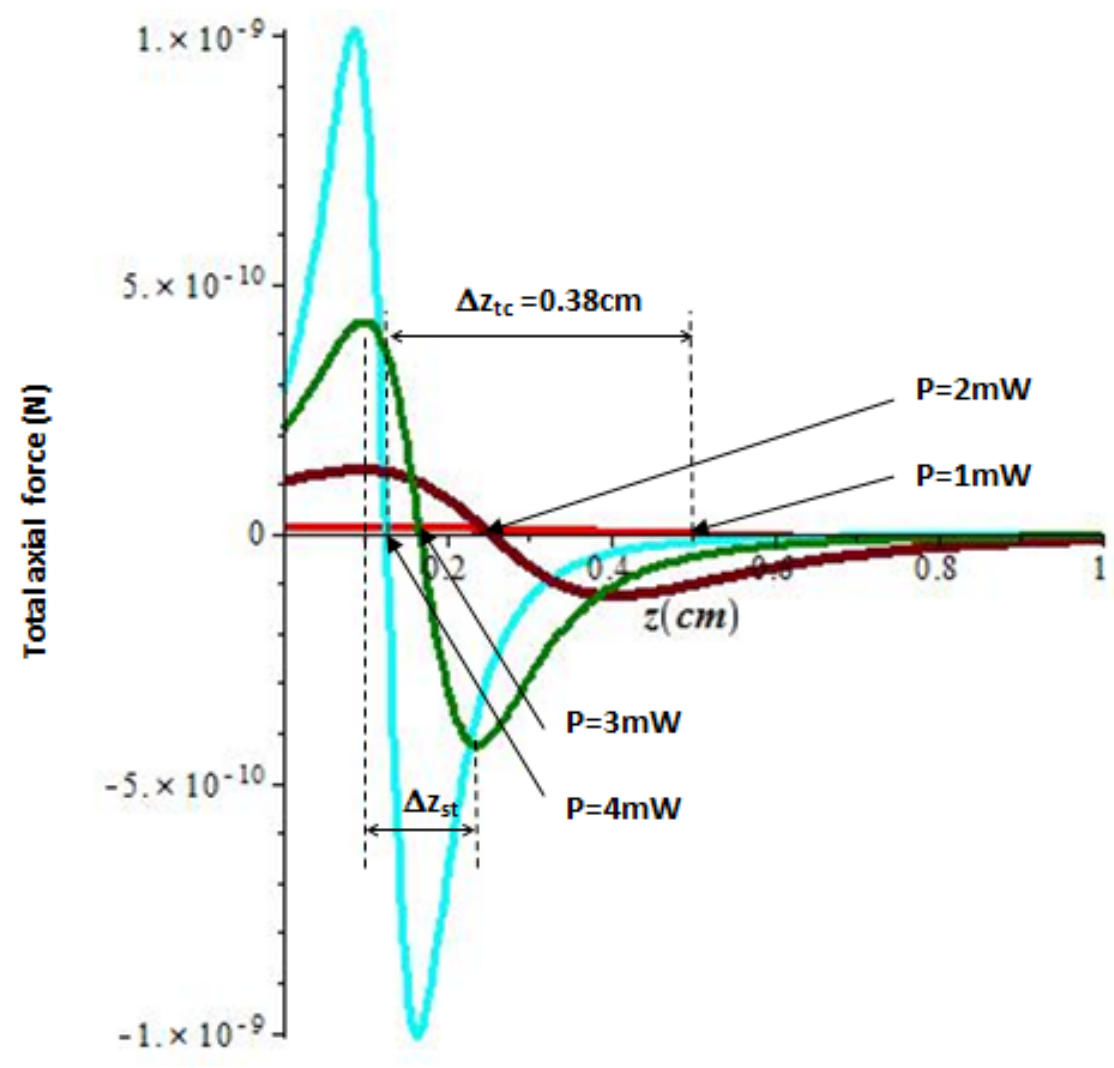

Fig. 10. Distribution of longitudinal force along laser beam axis [28].

We can see that OTE of LOT depends not on the laser power, but it is opposite to NOTOD. If $P \leq 2.15 \mathrm{~mW}$ the OTE of NOTOD is smaller than that of LOT, but it is opposite if $P \geq 2.15 \mathrm{~mW}$. This property can be explained as that the nonlinear effect appears not in layer of Acid Blue, means the nonlinear lens appears not until the average laser power is higher than certain threshold. Consequently, the NA of optical system is smaller than that of microscope objective used in LOT. And then, the average power increases larger than threshold that leads the beam waist as well as Rayleigh distance reduces, consequences increasing of intensity gradient of laser beam. It results in the increase of the optical forces and then enhancement of OTE of NOTOD, meanwhile, OTE 
of LOT is constant, as seen from Eqs. (1), (3) and (5): the OTE of LOT can be derived as

$$
\begin{aligned}
Q_{\rho, \text { max }} & =\frac{c F_{\text {grd }, \rho}^{\text {peak }}}{n_{s f} P}=\frac{c}{n_{s f}} \frac{\alpha\left\{\frac{2}{W_{0}\left[1+\left(\frac{z}{z_{0}}\right)^{2}\right]}\right\} I_{0} \exp \left(-\frac{1}{\left(1+\left(z / z_{0}\right)^{2}\right)}\right)}{\frac{\pi W_{0}^{2} I_{0}}{2}} \\
= & \frac{4 c \alpha}{\pi n_{s f} W_{0}^{3}\left[1+\left(\frac{z}{z_{0}}\right)^{2}\right]} \exp \left(-\frac{1}{\left(1+\left(z / z_{0}\right)^{2}\right)}\right)=\text { const }
\end{aligned}
$$

here $F_{g r d, \rho}^{p e a k}$ is fitted at $\rho=W_{0}$. That is similar for $Q_{z, \max }$, which is independent from the average laser power.

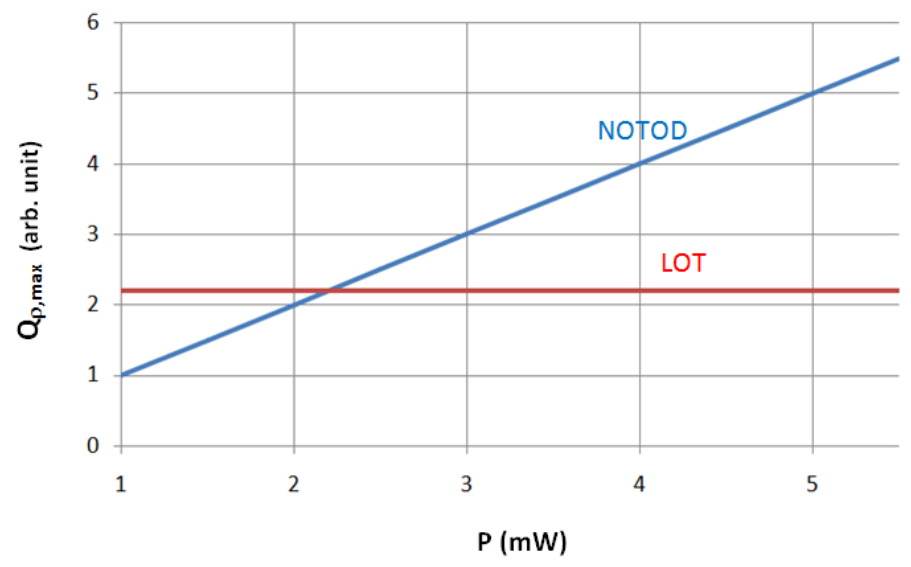

(a)

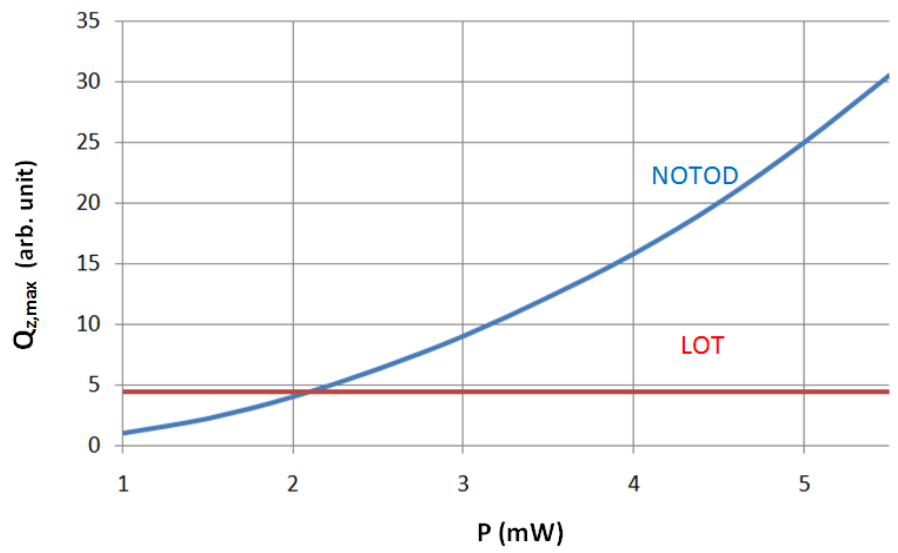

(b)

Fig. 11. $\left|Q_{\rho, \max }\right|$ (a) and $\left|Q_{z, \max }\right|$ (b) vs. $P$. 


\section{FLEXIBILITY OF CHOOSING NOTOD FOR DNA MOLECULES}

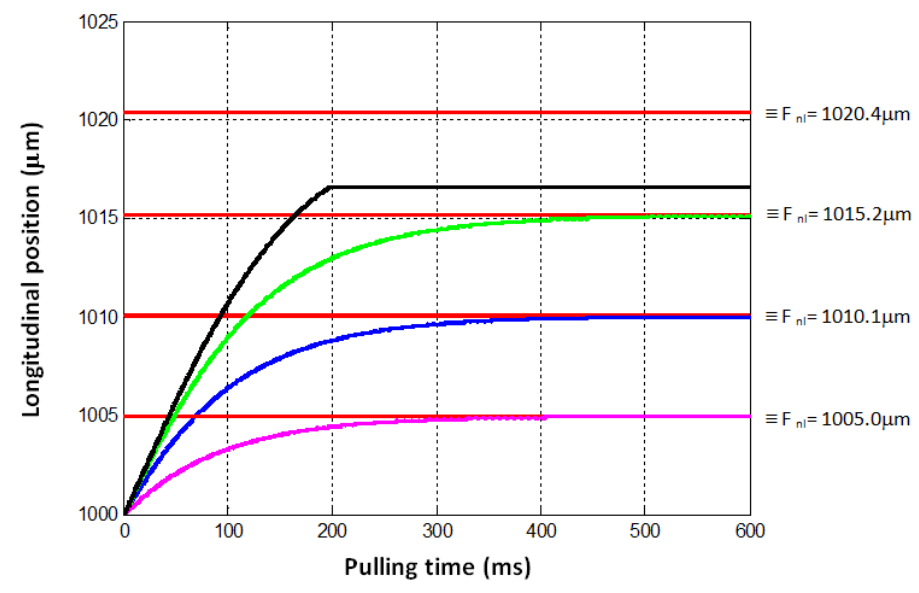

(a)

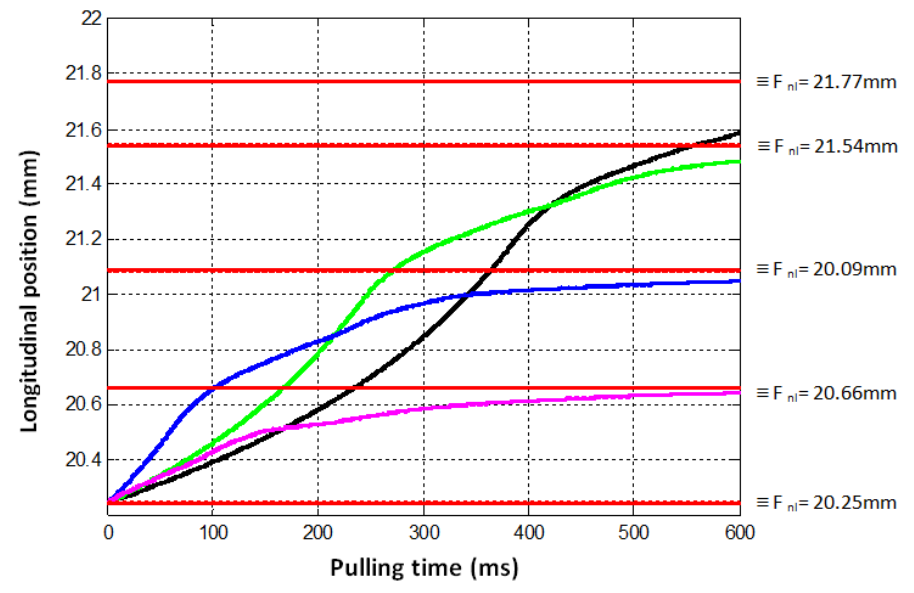

(b)

Fig. 12. (Color online) Bead position-pulling time characteristics of polystyrene bead linked to $\lambda$-phage DNA with peak intensities (down-up) of $I_{0}=0.995 \times 10^{5} \mathrm{Wcm}^{-2}$ (magenta), $I_{0}=0.99 \times 10^{5} \mathrm{Wcm}^{-2}$ (blue), $I_{0}=0.985 \times 10^{5} \mathrm{Wcm}^{-2}$ (green), $I_{0}=0.98 \times$ $10^{5} \mathrm{Wcm}^{-2}$ (black). b) Bead position-pulling time characteristics of polystyrene bead linked to BEC with peak intensities (down-up) of $I_{0}=0.98 \times 10^{5} \mathrm{Wcm}^{-2}$ (magenta), $I_{0}=0.96 \times 10^{5} \mathrm{Wcm}^{-2}$ (blue), $I_{0}=0.94 \times 10^{5} \mathrm{Wcm}^{-2}$ (green), $I_{0}=0.93 \times 10^{5} \mathrm{Wcm}^{-2}$ (black).

The main purpose of using OT in biophysics is to stretch DNA molecule in the extreme stretched state in which stretched length is comparable to contour length. As we know, the different kind of DNA has own contour length, may be from some nanometers (plasmid DNA with 
contour length of $3 \mathrm{~nm}[42]$ ) to some millimeters (Bacteria Escherichia Coli (BEC) with contour length of $1.5 \mathrm{~mm}$ [43]). It is very difficult to use a linear OT to stretch all of DNA kind by electrooptical or electro-mechanical methods [1,9-13]. But the difficulty can be overcome when using NOTOD. As shown in Sec. 5, the stable site of trapped bead can be controlled with high OTE by tune of laser power. It is favorable to use NOTOD for stretching of DNA molecules. By MATLAB software using finite element method $[6,38,40]$, the general Langevin equation as shown in Eq. (13) is solved, consequently, we observed numerically the position-pulling time characteristics of polystyrene bead ( $a=200 \mathrm{~nm}, n_{p}=1.5$ ) linked to $\lambda$-phage $\lambda I_{0}=0.995 \times 10^{5} \mathrm{Wcm}^{-2}$, $I_{0}=0.99 \times 10^{5} \mathrm{Wcm}^{-2}, I_{0}=0.98 \times 10^{5} \mathrm{Wcm}^{-2}, I_{0}=0.98 \times 10^{5} \mathrm{Wcm}^{-2}, I_{0}=0.96 \times 10^{5} \mathrm{Wcm}^{-2}$, $I_{0}=0.94 \times 10^{5} \mathrm{Wcm}^{-2}$ DNA with different peak intensities of laser beam with $W_{0}=0.01 \mathrm{~cm}$. From Fig. 12a, we can see that if the intensity is chosen of $I_{0}=1 \times 10^{5} \mathrm{Wcm}^{-2}$ and the longitudinal force hold trapped bead at beginning position of $1000 \mu \mathrm{m}$ from the output surface of Acid blue layer, then, by lowering the intensity, the trapped bead moves far away from the output surface of Acid blue layer. The main result is that when intensity is tuned an amount of $2 \mathrm{~kW} / \mathrm{cm}^{2}$ (black curve), the trapped moves a distance about of $16.5 \mu \mathrm{m}$, which is comparable to the contour length of $\lambda$-phage DNA molecule [42]. This situation is similar to the case of BEC in Fig. 12b. By slightly changing NA of low NA microscope objective, so that the beam waist is $W_{0}=0.045 \mathrm{~cm}$. With fixed beginning intensity $I_{0}=1 \times 10^{5} \mathrm{Wcm}^{-2}$ the bead hold at the longitudinal position of $20.25 \mathrm{~mm}$ from output surface of Acid blue layer, and then by tuning intensity lower to pull trapped bead farther. When intensity is tuned lower an amount of $7 \mathrm{~kW} / \mathrm{cm}^{2}$ the motion distance of trapped bead linked to BEC is about $1.4 \mathrm{~mm}$ comparable to the contour length. The aboveobtained results show that by finely tuning intensity of laser, the NOTOD can be used to stretch DNA molecule with the different contour length limited in certain long interval. That shows the flexibility of NOTOD.

\section{CONCLUSION}

The influence of Kerr effect on the properties of optical tweezers is resumed. The optical trap efficiency is higher for Kerr particle, since the index ratio (m) is enhanced, i.e. particle's refractive index is raised up by laser intensity. Even though particles have refractive index smaller than that of linear surrounding fluid, it is not necessary to use the hollow beam. The optical tweezers using Kerr medium (NOT) will be a tool to control linear particle in space by tuning laser power, which called the all-optical method although it can reduce optical trap efficiency, since the index radio is reduced by the laser intensity. The weakness of optical tweezers using Kerr medium is overcome by NOTOD, which uses organic dye as nonlinear lens replacing the nonlinear surrounding fluid. The proposed NOTOD is not only compact, having high optical efficiency, but also a flexible tool for optical method to control trapped bead in space and to stretch different kind of DNA molecules. Using organic dye with high nonlinearity, the optical trap efficiency of NOTOD is high enough, which can trap nanoparticle [21,22] and stretch DNA molecule [44-47], instead of using ultrafast pulsed laser.

The organic dye is not only in liquid phase, but also can be prepared as thin layer. Moreover, its linear index [28] is comparable to that of immersion oil, which is used to enhance NA of microscope objective [48]. So thin layer of organic dye could be used as immersion oil to design nonlinear immersion microscope objective for OT. We hope our suggestions may become advantageous in the going time. 


\section{ACKNOWLEDGMENT}

This research is funded by Vietnam National Foundation for Science and Technology Development (NAFOSTED) under grant number 103.03-2018.342.

\section{REFERENCES}

[1] J. H. G. Huisstede, Scanning probe optical tweezers: A new tool to study dna-protein interactions, Ph.D. thesis, University of Twente, 2006.

[2] P. Jing, J. Wu, G. W. Liu, E. G. Keeler, S. H. Pun and L. Y. Lin, Scientific reports 6 (2016) 19924.

[3] A. Ashkin, Physical review letters 24 (1970) 156.

[4] M. P. MacDonald, L. Paterson, W. Sibbett, K. Dholakia and P. E. Bryant, Optics Letters 26 (2001) 863.

[5] D. Min, M. A. Arbing, R. E. Jefferson and J. U. Bowie, Protein Science 25 (2016) 1535.

[6] T. T. Dinh, K. D. Quoc, K. B. Xuan and Q. H. Quang, Optical and Quantum Electronics 48 (2016) 561.

[7] M. F. Duarte, M. A. Davenport, D. Takhar, J. N. Laska, T. Sun, K. F. Kelly and R. G. Baraniuk, IEEE signal processing magazine 25 (2008) 83.

[8] K. C. Neuman and S. M. Block, Review of scientific instruments 75 (2004) 2787.

[9] G. Römer and P. Bechtold, Physics procedia 56 (2014) 29.

[10] E. R. Dufresne, G. C. Spalding, M. T. Dearing, S. A. Sheets and D. G. Grier, Review of Scientific Instruments 72 (2001) 1810.

[11] Y. Tanaka, H. Kawada, S. Tsutsui, M. Ishikawa and H. Kitajima, Optics express 17 (2009) 24102.

[12] D. Min, M. A. Arbing, R. E. Jefferson and J. U. Bowie, Protein Science 25 (2016) 1535.

[13] Y. Hao, C. Canavan, S. S. Taylor and R. A. Maillard, Scientific reports 7 (2017) 10843.

[14] H. Van Nam, C. T. Le and H. Q. Quy, Comm. Phys. 23 (2013) 155.

[15] Q. Q. Ho et al., J. Phys. Scien. and Appl. 2 (2012) 301.

[16] H. Okamoto, Y. Jiang and T. Narushuima, Progress in electromagnetic research symposium, PIERS, 2016.

[17] T. T. Doan, K. D. Quoc and Q. H. Quang, Optical and Quantum Electronics 50 (2018) 51.

[18] M. Weber, Crc handbook of optical materials, 2003.

[19] M. Jahja, On nonlinear optical constants of polystyrene, International Symposium on Modern Optics and Its Applications, 2011.

[20] K. Tanaka, Journal of Materials Science: Materials in Electronics (2005) 633.

[21] A. Devi and A. K. De, Optics Express 24 (2016) 21485.

[22] Y. Jiang, T. Narushima and H. Okamoto, Nature Physics 6 (2010) 1005.

[23] Q. Wu, S. Chang and X. Huang, Flexible and highly effective trapping particles of optical tweezers in nonlinear optical imaging, Frontiers in Optics, Optical Society of America, 2018, pp. JW3A-119.

[24] H. Q. Quy and T. B. Tru, Comm. Phys. 6 (1996) 23.

[25] E. Koushki, A. Farzaneh and S. Mousavi, Applied Physics B 99 (2010) 565.

[26] R. R. Krishnamurthy and R. Alkondan, Optica Applicata 40 (2010) 187.

[27] S. Jeyaram and T. Geethakrishnan, Optics \& Laser Technology 89 (2017) 179.

[28] L. T. Nguyen, N. T. Hong, C. T. B. Thi and A. Q. Le, Journal of Nonlinear Optical Physics \& Materials 23 (2014) 1450020.

[29] Q. H. Quang, T. T. Doan, T. D. Quoc and T. N. Manh, Optics Communications 421 (2018) 94.

[30] H. Q. Quy, D. Q. Tuan, T. D. Thanh and N. M. Thang, Optics Communications 427 (2018) 341.

[31] D. Q. Tuan, N. T. T. Tam, H. T. Loan and N. T. Cam, Journal of Military Science and Technology: Special Issue 57A (2018) 97.

[32] J. Du, C.-H. Yuen, X. Li, K. Ding, G. Du, Z. Lin, C. Chan and J. Ng, Scientific reports 7 (2017) 18042.

[33] T. Godazgar, R. Shokri and S. N. S. Reihani, Optics letters 36 (2011) 3284.

[34] H. Q. Quy, M. Van Luu, H. D. Hai and D. Zhuang, Chinese Optics Letters 8 (2010) 332.

[35] A. Ashkin, Biophysical journal 61 (1992) 569.

[36] V. Hoang, T. Cao and Q. Ho, Int. J. Eng. Innov. Techno. 3 (2013) 134.

[37] B. Saleh and M. Teich, Fundamentals of photonics [electronic resource], Wiley, 1991.

[38] G. Volpe and G. Volpe, American Journal of Physics 81 (2013) 224. 
[39] P. Mangeol, D. Côte, T. Bizebard, O. Legrand and U. Bockelmann, Eur. Phys. J. E 19 (2006) 311.

[40] T. D. Trung, Control the tension of dna molecule in nonlinear solvent with optical tweezers, Ph.D. thesis, Vinh University, 2017.

[41] E. Koushki, A. Farzaneh and S. Mousavi, Applied Physics B 99 (2010) 565.

[42] D. T. Thai, V. L. Chu and Q. Q. Ho, IJEIT 3 (2014) 159.

[43] C. G. Baumann, V. A. Bloomfield, S. B. Smith, C. Bustamante, M. D. Wang and S. M. Block, Biophysical journal 78 (2000) 1965

[44] S. Bakshi, A. Siryaporn, M. Goulian and J. C. Weisshaar, Molecular microbiology 85 (2012) 21.

[45] A. Keloth, O. Anderson, D. Risbridger and L. Paterson, Micromachines 9 (2018) 434.

[46] F. Falleroni, V. Torre and D. Cojoc, Frontiers in cellular neuroscience 12 (2018) 130.

[47] J. Liu and Z. Li, Micromachines 9 (2018) 232.

[48] A. A. R. Neves, A. Fontes, C. L. Cesar, A. Camposeo, R. Cingolani and D. Pisignano, Physical review E 76 (2007) 061917. 